\title{
Volatility of Cryptocurrencies
}

\section{Volatilnost kriptovaluta}

\begin{abstract}
Many models have been developed to model, estimate and forecast financial time series volatility, amongst which are the most popular autoregressive conditional heteroscedasticity (ARCH) model introduced by Engle (1982) and generalized autoregressive conditional heteroscedasticity (GARCH) model introduced by Bollerslev (1986). The aim of this paper is to determine which type of ARCH/GARCH models can fit the best following cryptocurrencies: Ethereum, Neo, Ripple, Litecoin, Dash, Zcash and Dogecoin. It is found that the EGARCH model is the best fitted model for Ethereum, Zcash and Neo, PARCH model is the best fitted model for Ripple, while for Litecoin, Dash and Dogecoin it depends on the selected distribution and information criterion.
\end{abstract}

Keywords: cryptocurrency returns, heteroscedasticity, ARCH/GARCH models

JEL classification: $\mathrm{C} 3, \mathrm{C} 53, \mathrm{G} 11, \mathrm{G} 17$

\section{Sažetak}

Brojni modeli su razvijeni kako bi se modelirala, procijenila i predvidjela volatilnost financijskih vremenskih serija, među kojima su najpopularniji autoregresijski model uvjetne heteroskedastičnosti (ARCH) kojega je osmislio Engle (1982) i generalizirani model uvjetne heteroskedastičnosti (GARCH) kojega je osmislio Bollerslev (1986). Cilj ovog rada je odrediti koji oblik ARCH/GARCH modela najbolje odgovara sljedećim kriptovalutama: Ethereumu, Neu, Rippleu, Litecoinu, Dashu, Zcashu i Dogecoinu. Utvrđeno je kako EGARCH model najbolje odgovara Ethereumu, Zcashu i Neu, PARCH model najbolje odgovara Rippleu, dok za Litecoin, Dash i Dogecoin izbor najboljeg modela ovisi o odabranoj distribuciji i informacijskom kriteriju.

Ključne riječi: prinosi kriptovaluta, heteroskedastičnost,ARCH/GARCH modeli JEL klasifikacija: C3, C53, G11, G17

\section{Introduction}

There is an increasing importance of the cryptocurrency market over the last decade, especially Bitcoin, the most popular one, that started to rise in value after 2008 , as an alternative monetary system, first described by Nakamoto (2008). Furthermore, (Bariviera et al., 2017) mention cryptocurrencies decentralization, use of blockchain technology, and low transaction costs for international transfers as advantages, while on the other hand, shortcomings include difficulties in assessing their fundamental value which gives them the character of a speculative bubble, still marginal public acceptance, the possibility of a Ponzi scheme, lack of open savings accounts in cryptocurrencies, and non-existence of interest rates, as the rates of return. According to Symitsi \& Chalvatzis (2018), cryptocurrencies are a very popular decentralized transactional system based on blockchain technology, while Dhyrberg (2016) states that, according to official researches, most users use Bitcoin in the form of speculative investments, rather than a mean of payment.

\section{Branimir Cvitko Cicvarić}

Addiko Bank d.d.

E-mail: branimir.cicvaric@gmail.com

\section{Branimir Cvitko Cicvarić}

Addiko Bank d.d.

E-mail:branimir.cicvaric@gmail.com 
Cvitko Cicvarić, B.

Volatility of Cryptocurrencies
Looking at the historical speculative bubbles Bjordal \& Opdahl (2017) presented in their research list of nine most popular bubbles where, according to the combination of years around peak and multiple of the starting price, Bitcoin can be classified as the biggest bubble in history, even before tulip mania. Klein et al. (2018) state that the CFTC (Commodity Futures Trading Commission) has described virtual money in their publication as an asset, such as oil or gold. However, they believe that Bitcoin cannot be considered the new gold, although it shares some similarities with it, due to the dynamics of its volatility. Moreover, from a portfolio perspective, Bitcoin cannot serve as a safe haven, such as gold. According to Osterrieder \& Lorenz (2017) the volatility of Bitcoin returns was 6 times higher than the volatility of $\mathrm{G} 10$ currencies returns in the time period from 2013 until 2016, measured by valueat-risk and expected shortfall. By using quantile cross-spectral analysis Baumöhl (2019) examined the existence of the short-term, medium-term and long-term correlation between cryptocurrencies and some forex currencies. Looking at the results of the correlation analysis in the short term and the long term, Ripple was found as a cryptocurrency with the most beneficial diversification potential. In the medium term, many diversification pairs between cryptocurrencies and forex currencies were found, like Litecoin and Japanese yen or Ripple and Japanese yen. In the long term, correlation results also suggest diversification between these two groups of currencies in market turmoil times. Given that the issue of cryptocurrency volatility is of great importance for further research, this paper will examine and present empirical results for the volatility of 7 cryptocurrencies returns: Ethereum, Neo, Dash, Zcash, Litecoin, Dogecoin and Ripple. The structure of the paper is divided into 5 main sections, where after the topic introduction follows a brief literature overview on the volatility of cryptocurrency returns, after which description of the data analyzed, the methodology used, estimated results obtained and discussion will be presented.

\section{Brief overview of related literature}

Klaassen (2002) states that volatility in financial returns is one of the most important aspects when making investment decisions. For instance, exchange rate volatility is the basis for determining the price of currency derivatives (options), which are later used for hedging in risk management. Symitsi \& Chalvatzis (2018) examined correlation between cryptocurrency market and other different assets in order to determine hedging potentials. Based on the calculated correlations between cryptocurrency market and other assets some potential hedging opportunities were found.As discussed later, several model specifications of ARCH will be presented for which (Bošnjak et al., 2016, pp. 82) state that ,the main purpose of the autoregressive conditional heteroscedasticity $(\mathrm{ARCH})$ model is to estimate the conditional variance of a time series."

Stavroyiannis (2018) in his research presented the results of the GARCH $(1,1)$ model obtained on several cryptocurrencies analyzed. The GARCH term was significant for all time series observed, while the leverage effect was presented in Bitcoin and Litecoin returns. In their paper Chu et al. (2017) conducted a study of 12 different GARCH models on 7 leading cryptocurrencies in the period from 2014 until 2017. Among the 12 different GARCH models, according to the values of the following information criteria: AIC, AICc, BIC, HOC, and CAIC, IGARCH $(1,1)$ model with normal innovations proved to be the best fit for Bitcoin, Dash, Litecoin, MaidSafeCoin, and Monero, due to the lowest values obtained by the criteria. The GJRGARCH $(1,1)$ model with normal innovations gives the lowest values of the criteria for Dogecoin, while the GARCH $(1,1)$ model with normal innovations, after the same logic, is the best fitted model for Ripple. Analyzing volatility of the Bitcoin returns between 2016 and 2018 Katsiampa (2019) concluded that the best fitted model is AR-CGARCH.Analysis of the Bitcoin returns was also performed by Letra (2016) where is found that GARCH $(1,1)$ model is the best fitted model. Looking at the CRIX and its volatility Chen et al. (2016) empirically concluded that the TGARCH $(1,1)$ model is the best fitted model. By using GARCHMIDAS models, Conrad et al. (2018) proved that the volatilities of S\&P500 and VIX index have negative long term effect on the Bitcoin returns.

In order to determine asymmetric effects, Baur et al. (2018) made calculations for asymmetric GARCH models. Different assets and their volatilities have been analyzed in order to determine if 
Table 1 Results of descriptive statistics

\begin{tabular}{|l|c|c|c|c|c|c|c|c|}
\hline & Zcash & Ripple & Neo & Litecoin & Ethereum & Dogecoin & Dash \\
\hline Mean & -0.0043 & 0.001939 & 0.003095 & 0.001135 & 0.002978 & 0.001001 & 0.002935 \\
\hline Median & -0.00486 & -0.00287 & -0.00418 & 0.00000 & -0.00093 & -0.00367 & -0.002186 \\
\hline Maximum & 1.038948 & 1.027356 & 0.801166 & 0.828968 & 0.412337 & 1.166254 & 1.270565 \\
\hline Minimum & -1.26884 & -0.61627 & -0.52254 & -0.513925 & -1.30211 & -0.580427 & -0.467565 \\
\hline Std. Dev. & 0.09472 & 0.07669 & 0.103644 & 0.06673 & 0.076916 & 0.080144 & 0.080574 \\
\hline Skewness & -1.17151 & 2.015239 & 1.082589 & 1.740597 & -3.38534 & 2.184293 & 2.99293 \\
\hline Kurtosis & 58.46237 & 30.53093 & 13.4789 & 28.19509 & 68.36974 & 35.44931 & 45.04492 \\
\hline Jargue-Bera & 109652.3 & 65484.15 & 3897.61 & 57521.18 & 234488.2 & 85003.99 & 138426.7 \\
\hline Prob. & 0.0000 & 0.0000 & 0.0000 & 0.0000 & 0.0000 & 0.0000 & 0.0000 \\
\hline Obs. & 855 & 2031 & 818 & 2135 & 1304 & 1904 & 1843 \\
\hline
\end{tabular}

Source: own estimates (data available at: https://coinmarketcap.com/).

there exist some differences in the processes of volatility between Bitcoin returns and other asset returns. The empirical analysis confirmed that hypothesis and volatility processes were found to be different. Catania et al. (2018) emphasize that cryptocurrencies have much in common with other financial assets, such as asymmetric reactions. Furthermore, they believe that models used so far, for instance, the GARCH models, should be replaced by better alternatives, such as the Score Driven model, for which they give empirical evidence representing better future potentials in volatility modeling. Similar conclusion on alternative models can be found in research by Urquhart (2017) where is suggested to use HAR models, instead of traditional GARCH models for modeling volatility. In their research Henriques \& Sadorsky (2018), by using variants of asymmetric GARCH models, empirically examined that investors averse to risk would be willing to change natural gold with Bitcoin in their portfolios. Analysis of spillovers by Corbet et al. (2018) draws conclusions about the isolation of the cryptocurrency market from external market shocks, which suggests their possible use as a portfolio diversifier. According to research by Cheng (2018), portfolios with included Bitcoin have better performance than without it. Furthermore, according to Dhyrberg (2016), it is important to point out that once more than $2 \%$ of Bitcoin is included, portfolio returns will start to decline. The inclusion of $2 \%$ of Bitcoin significantly increases the annual return from the initial $10 \%$ to $17 \%$, while the addition of more than $2 \%$ of Bitcoin leads to decrease in return compared to the initial period. Thus, as a conclusion, it is imposed that Bitcoin has diversification potentials, however, to a certain extent.

\section{Research data}

The data span used as a modeling sample differs for each cryptocurrency analyzed, with the beginning at the emergence of each cryptocurrency until the end of March 2019. The returns analyzed are calculated as the first differences between natural logarithms of closed prices. As explained by Bošnjak et al. (2016), among others, when returns exhibit values of kurtosis greater than 3, which is attributed to the normal distribution, they can be described as leptokurtic. As can be seen in Table 1 for all time series observed kurtosis is greater than 3 , indicating that normality assumptions are not met, so, when modeling volatilities, two different distributions will be used: Student $t$ distribution and Generalized Error Distribution.

Returns for all observed cryptocurrencies are stationary time series which is proved by the obtained p-values from Table 2 (around 0.00) as the first necessary step towards modeling volatility. Based on the correlogram and the p-values obtained (around 0.00) in all observed time series some degree of autocorrelation and partial autocorrelation is found.

Table 2 Results of Augmented Dickey-Fuller stationarity test (ADF)

\begin{tabular}{|l|c|c|}
\hline & t statistic & P value \\
\hline Ethereum & -38.5209 & 0.0000 \\
\hline Dogecoin & -40.9412 & 0.0000 \\
\hline Dash & -43.57574 & 0.0001 \\
\hline Zcash & -36.89869 & 0.0000 \\
\hline Litecoin & -45.1984 & 0.0001 \\
\hline Ripple & -42.7149 & 0.0001 \\
\hline Neo & -28.607 & 0.0000 \\
\hline
\end{tabular}

Source: own estimates.
Cvitko Cicvarić, B.

Volatility of Cryptocurrencies 
Cvitko Cicvarić, B.

Volatility of Cryptocurrencies

\section{Methodology}

Literature offered several model specifications to explain volatilities in financial time series while capturing volatility that often characterizes financial time series, especially volatility clustering (Bošnjak et al., 2016). The first model to be estimated is a baseline ARCH model introduced by Engle (1982) whose conditional variance equation is given by:

$$
\sigma^{2}=\omega+\alpha \epsilon_{t-1}^{2}
$$

The second model to be estimated is GARCH (1, 1) model introduced by Bollerslev (1986) whose conditional variance equation is given by:

$$
\sigma^{2}=\omega+\alpha \varepsilon_{t-1}^{2}+\beta \sigma_{t-1}^{2}
$$

Where, according to Bošnjak et al. (2016) first two terms are the same as in the baseline ARCH specification where term $\epsilon_{\mathrm{t}-1}^{2}$ captures volatility from the previous time period and the GARCH term $\sigma_{t}^{2}-1$ captures previous time period forecast variance in order to better capture volatility clustering.

The third model to be estimated is the TARCH model, introduced by Glosten et al. (1993) and (Zakoian, 1994), which represents an extension of the above-explained GARCH model in estimating asymmetric news impact that is described as a phenomenon of higher volatility response due to downward price movement compared to the equivalent upward movement $(\gamma>0)$. The TARCH specification for the conditional variance equation is given by:

$\sigma_{t}^{2}=\omega+\alpha \varepsilon_{t-1}^{2}+\gamma \varepsilon_{t-1}^{2} d_{t-1}+\beta \sigma_{t-1}^{2}$

Where $\gamma \varepsilon_{t-1}^{2} d_{t-1}$ refers to the leverage effect, and if $\gamma \neq 0$, according to Higgs \& Worthington (2005) effect of news will be asymmetric. The fourth model to be estimated is Exponential GARCH (EGARCH) specified by Nelson (1991) which represents an extension of GARCH specification that takes into account logarithmic values of conditional variance guaranteeing this way non-negativity of forecasts and exponentiality of leverage effects $(\gamma \mathrm{k}<0)$. Conditional variance equation is given by:

$$
\begin{gathered}
\log \left(\sigma_{t}^{2}\right)=\omega+\sum_{j=1}^{q} \beta_{j} \log \left(\sigma_{t-j}^{2}\right)+ \\
\sum_{i=1}^{p} \alpha_{i}\left|\frac{\varepsilon_{t-1}}{\sigma_{t-1}}\right|+\sum_{k=1}^{r} \gamma_{k}\left|\frac{\varepsilon_{t-k}}{\sigma_{t-k}}\right|
\end{gathered}
$$

The fifth model to be estimated is Power-ARCH (PARCH) specified by Ding et al. (1993) whose conditional variance equation is given by:

$$
\begin{gathered}
\sigma_{t}^{\delta}=\omega+\sum_{j=1}^{q} \beta_{j} \sigma_{t-j}^{\delta}+ \\
\sum_{i=1}^{p} \alpha\left(\left|\varepsilon_{t-1}\right|-\gamma \varepsilon_{t-1}\right)^{\delta}
\end{gathered}
$$

and, according to Bošnjak et al. (2016), this model is used to generalize the transformation of error terms in the models. 


\section{Empirical results and discussion}

Table 3 Parameter estimates for Ethereum - Student t distribution

\begin{tabular}{|c|c|c|c|c|c|}
\hline Parameter & ARCH (1) & $\operatorname{GARCH}(1,1)$ & TARCH & EGARCH & PARCH \\
\hline \multirow[b]{2}{*}{$\omega$} & 0.004864 & 0.000352 & 0.000353 & -0.674688 & 0.005051 \\
\hline & $(0.0103)$ & $(0.0007)$ & $(0.0007)$ & $(0.0000)$ & $(0.1726)$ \\
\hline \multirow[b]{2}{*}{$a$} & 1.028189 & 0.378904 & 0.368148 & 0.449534 & 0.280678 \\
\hline & $(0.0231)$ & $(0.0002)$ & $(0.0004)$ & $(0.0000)$ & $(0.0000)$ \\
\hline \multirow{2}{*}{$\beta$} & & 0.697933 & 0.696707 & 0.927487 & 0.759015 \\
\hline & & $(0.0000)$ & $(0.0000)$ & $(0.0000)$ & $(0.0000)$ \\
\hline \multirow[b]{2}{*}{ Y } & & & 0.031124 & 0.003766 & 0.009737 \\
\hline & & & $(0.7380)$ & $(0.9080)$ & $(0.9024)$ \\
\hline \multirow{2}{*}{$\delta$} & & & & & 1.003035 \\
\hline & & & & & $(0.0000)$ \\
\hline ARCH - LM test & $(0.0616)$ & $(0.4420)$ & $(0.4405)$ & $(0.7175)$ & $(0.7233)$ \\
\hline $\mathrm{AlC}$ & -2844467 & -2912111 & -2910671 & -2917901 & -2915323 \\
\hline SIC & -2832558 & -2896232 & -2890823 & -2898053 & -2891505 \\
\hline Obs & 1304 & 1304 & 1304 & 1304 & 1304 \\
\hline
\end{tabular}

Source: own estimates.

Table 4 Parameter estimates for Ethereum - Generalized Error Distribution

\begin{tabular}{|l|c|c|c|c|c|}
\hline \multicolumn{1}{|c|}{ Parameter } & ARCH (1) & GARCH (1,1) & TARCH & EGARCH & PARCH \\
\hline$\omega$ & 0.002877 & 0.000347 & 0.000347 & -0.733614 & 0.007146 \\
\hline$a$ & $(0.0000)$ & $(0.0000)$ & $(0.0000)$ & $(0.0000)$ & $(0.2339)$ \\
\hline$\beta$ & 0.500554 & 0.263570 & 0.263980 & 0.371765 & 0.217912 \\
\hline Y & $(0.0000)$ & $(0.0000)$ & $(0.0000)$ & $(0.0000)$ & $(0.0000)$ \\
\hline Y & & 0.689525 & 0.689599 & 0.916668 & 0.759638 \\
\hline$\delta$ & & $(0.0000)$ & $(0.0000)$ & $(0.0000)$ & $(0.0000)$ \\
\hline ARCH - LM test & & & -0.001153 & 0.012331 & -0.029360 \\
\hline AIC & & & $(0.9873)$ & $(0.6942)$ & $(0.7639)$ \\
\hline SIC & & & & & 0.903057 \\
\hline Obs & & & & $(0.0010)$ \\
\hline
\end{tabular}

Source: own estimates.

Table 5 Parameter estimates for Neo - Student t distribution

\begin{tabular}{|l|c|c|c|c|c|}
\hline \multicolumn{1}{|c|}{ Parameter } & ARCH (1) & GARCH (1,1) & TARCH & EGARCH & PARCH \\
\hline$\omega$ & 0.008643 & 0.000383 & 0.000552 & -0.633627 & 0.004905 \\
\hline & $(0.0015)$ & $(0.01689$ & $(0.0075)$ & $(0.0000)$ & $(0.2721)$ \\
\hline$a$ & 0.588814 & 0.185198 & 0.250465 & 0.387426 & 0.213798 \\
\hline$\beta$ & $(0.0105)$ & $(0.0015)$ & $(0.0019)$ & $(0.0000)$ & $(0.0000)$ \\
\hline Y & & 0.829862 & 0.798090 & 0.918480 & 0.799253 \\
\hline$\delta$ & & $(0.0000)$ & $(0.0000)$ & $(0.0000)$ & $(0.0000)$ \\
\hline ARCH - LM test & & & -0.106651 & 0.084295 & -0.237821 \\
\hline AIC & & & $(0.1534)$ & $(0.0437)$ & $(0.0606)$ \\
\hline SIC & & & & 1.132248 \\
\hline Obs & & & & $(0.0008)$ \\
\hline
\end{tabular}

Source: own estimates. 
Cvitko Cicvarić, B.

Volatility of Cryptocurrencies
Table 6 Parameter estimates for Neo - Generalized Error Distribution

\begin{tabular}{|c|c|c|c|c|c|}
\hline Parameter & ARCH (1) & $\operatorname{GARCH}(1,1)$ & TARCH & EGARCH & PARCH \\
\hline \multirow[b]{2}{*}{$\omega$} & 0.006014 & 0.000277 & 0.000359 & -0.708230 & 0.006487 \\
\hline & $(0.0000)$ & $(0.0057)$ & $(0.0027)$ & $(0.0000)$ & $(0.3393)$ \\
\hline \multirow[b]{2}{*}{$a$} & 0.403646 & 0.134596 & 0.171076 & 0.345437 & 0.200996 \\
\hline & $(0.0001)$ & $(0.0000)$ & $(0.0000)$ & $(0.0000)$ & $(0.0000)$ \\
\hline \multirow{2}{*}{$\beta$} & & 0.842841 & 0.827821 & 0.905820 & 0.760710 \\
\hline & & $(0.0000)$ & $(0.0000)$ & $(0.0000)$ & $(0.0000)$ \\
\hline \multirow[b]{2}{*}{ Y } & & & -0.071810 & 0.087883 & -0.309594 \\
\hline & & & $(0.1304)$ & $(0.0217)$ & $(0.0218)$ \\
\hline \multirow[b]{2}{*}{$\delta$} & & & & & 1.117776 \\
\hline & & & & & $(0.0033)$ \\
\hline ARCH - LM test & $(0.6023)$ & $(0.2281)$ & $(0.4329)$ & $(0.8692)$ & $(0.5823)$ \\
\hline AIC & -2156304 & -2204533 & -2204662 & -2213349 & -2205712 \\
\hline SIC & -2139025 & -2181494 & -2175863 & -2184550 & -2171154 \\
\hline Obs & 818 & 818 & 818 & 818 & 818 \\
\hline
\end{tabular}

Source: own estimates.

Table 7 Parameter estimates for Ripple - Student t distribution

\begin{tabular}{|c|c|c|c|c|c|}
\hline Parameter & ARCH (1) & $\operatorname{GARCH}(1,1)$ & TARCH & EGARCH & PARCH \\
\hline \multirow[b]{2}{*}{$\omega$} & 1.136212 & 0.000359 & 0.000373 & -0.902562 & 0.008416 \\
\hline & $(0.9956)$ & $(0.0180)$ & $(0.0164)$ & $(0.0000)$ & $(0.0296)$ \\
\hline \multirow[b]{2}{*}{$a$} & 803.5835 & 1.046327 & 1.118940 & 0.761157 & 0.511490 \\
\hline & $(0.9956)$ & $(0.0176)$ & $(0.0175)$ & $(0.0000)$ & $(0.0000)$ \\
\hline \multirow{2}{*}{$\beta$} & & 0.633791 & 0.626340 & 0.893944 & 0.681877 \\
\hline & & $(0.0000)$ & $(0.0000)$ & $(0.0000)$ & $(0.0000)$ \\
\hline \multirow[b]{2}{*}{ Y } & & & -0.121532 & 0.017426 & -0.096004 \\
\hline & & & $(0.5338)$ & $(0.6769)$ & $(0.1095)$ \\
\hline \multirow{2}{*}{$\delta$} & & & & & 0.875615 \\
\hline & & & & & $(0.0000)$ \\
\hline ARCH - LM test & $(0.4921)$ & $(0.6359)$ & $(0.6049)$ & $(0.7540)$ & $(0.7885)$ \\
\hline AIC & -3157823 & -3238784 & -3238011 & -3234649 & -3248903 \\
\hline SIC & -3149524 & -3227718 & -3224179 & -3220817 & -3232305 \\
\hline Obs & 2031 & 2031 & 2031 & 2031 & 2031 \\
\hline
\end{tabular}

Source: own estimates.

Table 8 Parameter estimates for Ripple - Generalized Error Distribution

\begin{tabular}{|c|c|c|c|c|c|}
\hline Parameter & ARCH (1) & $\operatorname{GARCH}(1,1)$ & TARCH & EGARCH & PARCH \\
\hline \multirow[b]{2}{*}{$\omega$} & 0.001880 & 0.000232 & 0.000267 & -1.071369 & 0.006736 \\
\hline & $(0.0000)$ & $(0.0000)$ & $(0.0000)$ & $(0.0000)$ & $(0.0500)$ \\
\hline \multirow[b]{2}{*}{$a$} & 1.111644 & 0.429984 & 0.529365 & 0.528116 & 0.379927 \\
\hline & $(0.0000)$ & $(0.0000)$ & $(0.0000)$ & $(0.0000)$ & $(0.0000)$ \\
\hline \multirow{2}{*}{$\beta$} & & 0.631798 & 0.601831 & 0.876036 & 0.659441 \\
\hline & & $(0.0000)$ & $(0.0000)$ & $(0.0000)$ & $(0.0000)$ \\
\hline \multirow[b]{2}{*}{$\gamma$} & & & -0.128501 & 0.041976 & -0.137739 \\
\hline & & & $(0.1625)$ & $(0.1750)$ & $(0.0294)$ \\
\hline \multirow{2}{*}{$\delta$} & & & & & 0.944708 \\
\hline & & & & & $(0.0000)$ \\
\hline ARCH - LM test & $(0.5148)$ & $(0.6440)$ & $(0.5460)$ & (0.7121) & $(0.7256)$ \\
\hline AIC & -3142541 & -3223695 & -3223718 & -3219564 & -3231337 \\
\hline SIC & -3134242 & -3212629 & -3209886 & -3205732 & -3214739 \\
\hline Obs & 2031 & 2031 & 2031 & 2031 & 2031 \\
\hline
\end{tabular}

Source: own estimates. 
Table 9 Parameter estimates for Litecoin - Student t distribution

\begin{tabular}{|c|c|c|c|c|c|}
\hline Parameter & ARCH (1) & $\operatorname{GARCH}(1,1)$ & TARCH & EGARCH & PARCH \\
\hline \multirow[b]{2}{*}{$\omega$} & 4.137633 & 5.49E-05 & $5.22 \mathrm{E}-05$ & -0.227550 & 0.000864 \\
\hline & $(0.9989)$ & $(0.2005)$ & $(0.2034)$ & $(0.0000)$ & $(0.1475)$ \\
\hline \multirow[b]{2}{*}{$a$} & 2510.093 & 0.638148 & 0.700978 & 0.411680 & 0.342463 \\
\hline & $(0.9989)$ & $(0.1800)$ & $(0.1838)$ & $(0.0000)$ & $(0.0011)$ \\
\hline \multirow{2}{*}{$\beta$} & & 0.863923 & 0.867478 & 0.988543 & 0.867929 \\
\hline & & $(0.0000)$ & $(0.0000)$ & $(0.0000)$ & $(0.0000)$ \\
\hline \multirow[b]{2}{*}{$Y$} & & & -0.163945 & 0.004967 & -0.059325 \\
\hline & & & $(0.3480)$ & $(0.8480)$ & $(0.3924)$ \\
\hline \multirow{2}{*}{$\delta$} & & & & & 1.026197 \\
\hline & & & & & $(0.0000)$ \\
\hline ARCH - LM test & $(0.2558)$ & $(0.7301)$ & $(0.7223)$ & $(0.7761)$ & $(0.7835)$ \\
\hline $\mathrm{AlC}$ & -3360921 & -3509626 & -3509524 & -3516027 & -3519640 \\
\hline SIC & -3352956 & -3499006 & -3496249 & -3502752 & -3503710 \\
\hline Obs & 2135 & 2135 & 2135 & 2135 & 2135 \\
\hline
\end{tabular}

Source: own estimates.

Table 10 Parameter estimates for Litecoin - Generalized Error Distribution

\begin{tabular}{|c|c|c|c|c|c|}
\hline Parameter & $\operatorname{ARCH}(1)$ & $\operatorname{GARCH}(1,1)$ & TARCH & EGARCH & PARCH \\
\hline \multirow{2}{*}{ 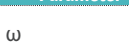 } & 0.001970 & 3.40E-05 & $3.33 \mathrm{E}-05$ & -0.273712 & 0.000851 \\
\hline & $(0.0000)$ & $(0.0003)$ & $(0.0004)$ & $(0.0000)$ & $(0.1646)$ \\
\hline \multirow{2}{*}{$a$} & 0.898465 & 0.139006 & 0.157808 & 0.239771 & 0.162529 \\
\hline & $(0.0000)$ & $(0.0000)$ & $(0.0000)$ & $(0.0000)$ & $(0.0000)$ \\
\hline \multirow{2}{*}{$\beta$} & & 0.877667 & 0.880171 & 0.980075 & 0.880726 \\
\hline & & $(0.0000)$ & $(0.0000)$ & $(0.0000)$ & $(0.0000)$ \\
\hline \multirow[b]{2}{*}{ Y } & & & -0.044545 & 0.016739 & -0.118205 \\
\hline & & & $(0.1875)$ & (0.3619) & $(0.1575)$ \\
\hline \multirow[b]{2}{*}{$\delta$} & & & & & 1.052125 \\
\hline & & & & & $(0.0000)$ \\
\hline ARCH - LM test & $(0.3153)$ & $(0.7313)$ & (0.7287) & $(0.7920)$ & $(0.7959)$ \\
\hline $\mathrm{AIC}$ & -3369249 & -3476822 & -3476902 & -3483152 & -3483160 \\
\hline SIC & -3361284 & -3466202 & -3463627 & -3469877 & -3467230 \\
\hline Obs & 2135 & 2135 & 2135 & 2135 & 2135 \\
\hline
\end{tabular}

Source: own estimates.

Table 11 Parameter estimates for Zcash - Student $t$ distribution

\begin{tabular}{|c|c|c|c|c|c|}
\hline Parameter & $\operatorname{ARCH}(1)$ & $\operatorname{GARCH}(1,1)$ & TARCH & EGARCH & PARCH \\
\hline \multirow[b]{2}{*}{$\omega$} & 0.004260 & 0.000278 & 0.000275 & -0.391431 & 0.003743 \\
\hline & $(0.0000)$ & $(0.0030)$ & $(0.0037)$ & $(0.0000)$ & $(0.3782)$ \\
\hline \multirow[b]{2}{*}{$a$} & 0.509502 & 0.141497 & 0.120215 & 0.229696 & 0.136065 \\
\hline & $(0.0005)$ & $(0.0005)$ & $(0.0033)$ & $(0.0000)$ & $(0.0001)$ \\
\hline \multirow{2}{*}{$\beta$} & & 0.823027 & 0.822405 & 0.957982 & 0.848747 \\
\hline & & $(0.0000)$ & $(0.0000)$ & $(0.0000)$ & $(0.0000)$ \\
\hline \multirow[b]{2}{*}{ Y } & & & 0.048414 & -0.011581 & -0.001188 \\
\hline & & & $(0.4129)$ & $(0.6960)$ & $(0.9930)$ \\
\hline \multirow[b]{2}{*}{$\delta$} & & & & & 1.012284 \\
\hline & & & & & $(0.0124)$ \\
\hline ARCH - LM test & $(0.1440)$ & (0.9379) & $(0.8542)$ & (0.5919) & $(0.6085)$ \\
\hline AlC & -2574537 & -2649163 & -2647813 & -2657402 & -2650046 \\
\hline SIC & -2557851 & -2626915 & -2620003 & -2629592 & -2616674 \\
\hline Obs & 855 & 855 & 855 & 855 & 855 \\
\hline
\end{tabular}

Source: own estimates. 
Cvitko Cicvarić, B.

Volatility of Cryptocurrencies
Table 12 Parameter estimates for Zcash - Generalized Error Distribution

\begin{tabular}{|c|c|c|c|c|c|}
\hline Parameter & ARCH (1) & $\operatorname{GARCH}(1,1)$ & TARCH & EGARCH & PARCH \\
\hline \multirow[b]{2}{*}{$\omega$} & 0.003577 & 0.000278 & 0.000278 & -0.403274 & 0.005650 \\
\hline & $(0.0000)$ & $(0.0028)$ & $(0.0032)$ & $(0.0001)$ & $(0.3895)$ \\
\hline \multirow[b]{2}{*}{ a } & 0.417017 & 0.122822 & 0.119168 & 0.208105 & 0.112937 \\
\hline & $(0.0000)$ & $(0.0001)$ & $(0.0006)$ & $(0.0001)$ & $(0.0004)$ \\
\hline \multirow{2}{*}{$\beta$} & & 0.818945 & 0.818937 & 0.954553 & 0.857908 \\
\hline & & $(0.0000)$ & $(0.0000)$ & $(0.0000)$ & $(0.0000)$ \\
\hline \multirow[b]{2}{*}{ Y } & & & 0.007910 & 0.007416 & -0.125074 \\
\hline & & & $(0.8747)$ & $(0.7870)$ & $(0.4317)$ \\
\hline \multirow{2}{*}{$\delta$} & & & & & 0.864287 \\
\hline & & & & & $(0.0401)$ \\
\hline ARCH - LM test & $(0.1532)$ & $(0.9253)$ & $(0.9114)$ & $(0.6094)$ & $(0.5186)$ \\
\hline $\mathrm{AIC}$ & -2577774 & -2642804 & -2640496 & -2650104 & -2643241 \\
\hline SIC & -2561088 & -2620556 & -2612686 & -2622294 & -2609869 \\
\hline Obs & 855 & 855 & 855 & 855 & 855 \\
\hline
\end{tabular}

Source: own estimates.

Table 13 Parameter estimates for Dash - Student t distribution

\begin{tabular}{|c|c|c|c|c|c|}
\hline Parameter & ARCH (1) & $\operatorname{GARCH}(1,1)$ & TARCH & EGARCH & PARCH \\
\hline \multirow{2}{*}{ Palrallinerer } & 0.004475 & 0.000246 & 0.000237 & -0.539900 & 0.001408 \\
\hline & $(0.0003)$ & $(0.0001)$ & $(0.0001)$ & $(0.0000)$ & $(0.1862)$ \\
\hline \multirow[b]{2}{*}{$a$} & 1.019023 & 0.274261 & 0.241084 & 0.398840 & 0.254907 \\
\hline & $(0.0012)$ & $(0.0000)$ & $(0.0000)$ & $(0.0000)$ & $(0.0000)$ \\
\hline \multirow[b]{2}{*}{$\beta$} & & 0.755903 & 0.756850 & 0.948287 & 0.790313 \\
\hline & & $(0.0000)$ & $(0.0000)$ & $(0.0000)$ & $(0.0000)$ \\
\hline \multirow[b]{2}{*}{$\gamma$} & & & 0.081039 & -0.023523 & 0.057281 \\
\hline & & & $(0.2129)$ & $(0.3719)$ & $(0.3672)$ \\
\hline \multirow[b]{2}{*}{$\delta$} & & & & & 1.330304 \\
\hline & & & & & $(0.0000)$ \\
\hline ARCH - LM test & $(0.2444)$ & $(0.8805)$ & $(0.8768)$ & $(0.4916)$ & $(0.4701)$ \\
\hline AIC & -2844850 & -2931459 & -2931461 & -2934460 & -2933303 \\
\hline SIC & -2835862 & -2919475 & -2916481 & -2919480 & -2915327 \\
\hline Obs & 1843 & 1843 & 1843 & 1843 & 1843 \\
\hline
\end{tabular}

Source: own estimates.

Table 14 Parameter estimates for Dash - Generalized Error Distribution

\begin{tabular}{|c|c|c|c|c|c|}
\hline Parameter & ARCH (1) & $\operatorname{GARCH}(1,1)$ & TARCH & EGARCH & PARCH \\
\hline \multirow[t]{2}{*}{  } & 0.002755 & 0.000195 & 0.000192 & -0.530650 & 0.001167 \\
\hline & $(0.0000)$ & $(0.0000)$ & $(0.0000)$ & $(0.0000)$ & $(0.2242)$ \\
\hline \multirow{2}{*}{ a } & 0.591983 & 0.205029 & 0.193971 & 0.341336 & 0.206345 \\
\hline & $(0.0000)$ & $(0.0000)$ & $(0.0000)$ & $(0.0000)$ & $(0.0000)$ \\
\hline \multirow[b]{2}{*}{$\beta$} & & 0.772080 & 0.772935 & 0.948892 & 0.800656 \\
\hline & & $(0.0000)$ & $(0.0000)$ & $(0.0000)$ & $(0.0000)$ \\
\hline \multirow[b]{2}{*}{ Y } & & & 0.025606 & -0.005045 & 0.012184 \\
\hline & & & $(0.5831)$ & $(0.8166)$ & $(0.8442)$ \\
\hline \multirow{2}{*}{$\delta$} & & & & & 1.353522 \\
\hline & & & & & $(0.0000)$ \\
\hline ARCH - LM test & $(0.2929)$ & $(0.6999)$ & $(0.7103)$ & (0.3411) & $(0.3377)$ \\
\hline AIC & -2838532 & -2919768 & -2918878 & -2921604 & -2920440 \\
\hline SIC & -2829544 & -2907784 & -2903898 & -2906624 & -2902465 \\
\hline Obs & 1843 & 1843 & 1843 & 1843 & 1843 \\
\hline
\end{tabular}

Source: own estimates. 
Table 15 Parameter estimates for Dogecoin - Student t distribution

\begin{tabular}{|c|c|c|c|c|c|}
\hline Parameter & ARCH (1) & $\operatorname{GARCH}(1,1)$ & TARCH & EGARCH & PARCH \\
\hline \multirow{2}{*}{ ralanteret } & 0.006193 & $8.80 \mathrm{E}-05$ & $8.92 \mathrm{E}-05$ & -0.472366 & 0.000730 \\
\hline & $(0.1934)$ & $(0.0004)$ & $(0.0004)$ & $(0.0000)$ & $(0.2068)$ \\
\hline \multirow[b]{2}{*}{ a } & 3.573057 & 0.316144 & 0.328800 & 0.430784 & 0.284433 \\
\hline & $(0.2068)$ & $(0.0000)$ & $(0.0000)$ & $(0.0000)$ & $(0.0000)$ \\
\hline \multirow[b]{2}{*}{$\beta$} & & 0.776175 & 0.775355 & 0.965231 & 0.799764 \\
\hline & & $(0.0000)$ & $(0.0000)$ & $(0.0000)$ & $(0.0000)$ \\
\hline \multirow[b]{2}{*}{ Y } & & & -0.024262 & 0.027671 & -0.068372 \\
\hline & & & $(0.7046)$ & $(0.2891)$ & $(0.2763)$ \\
\hline \multirow[b]{2}{*}{$\delta$} & & & & & 1.320519 \\
\hline & & & & & $(0.0000)$ \\
\hline ARCH - LM test & $(0.1483)$ & $(0.8235)$ & (0.8893) & $(0.5060)$ & $(0.4977)$ \\
\hline $\mathrm{AIC}$ & -3051434 & -3187922 & -3186962 & -3189149 & -3188707 \\
\hline SIC & -3042683 & -3176253 & -3172377 & -3174563 & -3171204 \\
\hline Obs & 1904 & 1904 & 1904 & 1904 & 1904 \\
\hline
\end{tabular}

Source: own estimates.

Table 16 Parameter estimates for Dogecoin - Generalized Error Distribution

\begin{tabular}{|c|c|c|c|c|c|}
\hline Parameter & ARCH (1) & $\operatorname{GARCH}(1,1)$ & TARCH & EGARCH & PARCH \\
\hline \multirow[t]{2}{*}{ 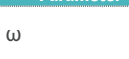 } & 0.002060 & $6.80 \mathrm{E}-05$ & $7.01 \mathrm{E}-05$ & -0.447107 & 0.000957 \\
\hline & $(0.0000)$ & $(0.0001)$ & $(0.0001)$ & $(0.0000)$ & $(0.2120)$ \\
\hline \multirow[b]{2}{*}{$a$} & 0.941961 & 0.220095 & 0.237502 & 0.353288 & 0.215503 \\
\hline & $(0.0000)$ & $(0.0000)$ & $(0.0000)$ & $(0.0000)$ & $(0.0000)$ \\
\hline \multirow{2}{*}{$\beta$} & & 0.797187 & 0.795399 & 0.966238 & 0.822097 \\
\hline & & $(0.0000)$ & $(0.0000)$ & $(0.0000)$ & $(0.0000)$ \\
\hline \multirow[b]{2}{*}{$\gamma$} & & & -0.032621 & 0.036247 & -0.115433 \\
\hline & & & $(0.4721)$ & $(0.1045)$ & $(0.0858)$ \\
\hline \multirow[b]{2}{*}{$\delta$} & & & & & 1.181726 \\
\hline & & & & & $(0.0000)$ \\
\hline ARCH - LM test & $(0.1802)$ & $(0.4902)$ & $(0.6056)$ & $(0.2757)$ & $(0.2034)$ \\
\hline $\mathrm{AIC}$ & -3048051 & -3176663 & -3175923 & -3180722 & -3178809 \\
\hline SIC & -3039300 & -3164994 & -3161338 & -3166137 & -3161306 \\
\hline Obs & 1904 & 1904 & 1904 & 1904 & 1904 \\
\hline
\end{tabular}

Source: own estimates.

When comparing Student $t$ distribution results with that of the Generalized Error Distribution some differences can be found, especially in the value of estimated coefficients. Results obtained through the Student $t$ distribution provide higher coefficient values for most of the estimated terms compared to the results of the Generalized Error Distribution. According to $\mathrm{p}$-values obtained from Q-Statistics, there is the existence of serial correlation among residuals in any estimated ARCH type model for Ethereum, Neo and Ripple (p-values above 0.05), while residuals in estimated ARCH type models of Dash, Dogecoin, Zcash and Litecoin do not suffer from serial correlation ( $p$-values around 0.00 ). For all models estimated, as a part of diagnostic checking, the ARCH LM test was performed, and no ARCH behavior was found in any of them (p-values above 0.05). Comparing AIC (Akaike
Information Criterion) and SC (Schwarz Criterion) for estimated volatility models it can be concluded that the EGARCH model is the best fitting model for Ethereum, $\mathrm{Neo}$ and Zcash since it minimizes values for the mentioned information criteria. Following the same rule, the PARCH model is the best fitted for Litecoin returns under Student $t$ distribution, while minimized value of AIC under Generalized Error Distribution suggests also PARCH model as the best fitted, and on the other hand, minimized SC value suggests EGARCH model. The same dilemma occurred when deciding which model is the best fit for Dash returns. Under assumptions of the Student t distribution, EGARCH model was found as the most appropriate, due to both information criteria, while under assumptions of the Generalized Error Distribution minimized value of AIC suggests also EGARCH model, but minimized value of SC suggests
Cvitko Cicvarić, B.

Volatility of Cryptocurrencies 
Cvitko Cicvarić, B.

Volatility of Cryptocurrencies
GARCH (1,1) model. Likewise, information criteria under Generalized Error Distribution suggest EGARCH as the best fitting model for Dogecoin, while under Student $\mathrm{t}$ distribution AIC also suggests EGARCH model, but SC suggests GARCH $(1,1)$ model. In the case of Ripple, information criteria under both distributions suggest PARCH as the best fitted model. When analyzing ARCH and GARCH terms in selected models one can find their statistical significance in all best fitting models, whose sum is greater than 1 in all cases. Looking at the term representing leverage effect no significant value was found, with the exception of Ripple under Generalized Error Distribution in the PARCH specification.

\section{Conclusion}

This paper investigates the Ethereum, Neo, Ripple,
Zcash, Dash, Dogecoin and Litecoin volatility of returns using GARCH volatility models. The time series are found to be stationary and characterized by leptokurtosis, so alternative distributions are used: Student t distribution and Generalized Error Distribution. Based on the Akaike Information Criterion (AIC) and Schwarz Criterion (SC) EGARCH model is the best fitting model for Ethereum, Neo and Zcash, while PARCH model is best fitted for Ripple. The dilemma on selecting the best fitting model occurred on the other cryptocurrencies, and depending on different information criterion and distribution assumptions, it can be either EGARCH or GARCH $(1,1)$ model for Dash, EGARCH or PARCH model for Litecoin, and EGARCH or GARCH $(1,1)$ model for Dogecoin. Statistical significance of leverage effect is found only for Ripple returns, under Generalized Error Distribution assumptions.

\section{References}

Bariviera, A. F., Basgall, M. J., Hasperué, W., Naiouf, M. (2017) Some stylized facts of the Bitcoin market. Physica A: Statistical Mechanics and its Applications, 484: 82-90. doi:10.1016/j.physa.2017.04.159.

Baumöhl, E. (2019) Are cryptocurrencies connected to forex? A quantile cross-spectral approach. Finance Research Letters, 29: 363-372. doi:10.1016/j.frl.2018.09.002.

Baur, D. G., Dimpfl, T., Kuck, K. (2018) Bitcoin, gold and the US dollar-A replication and extension. Finance Research Letters, 25:103-110. doi:10.1016/.j.fr.2017.10.012.

Bjordal, A., Opdahl, E. (2017) Portfolio optimization in the cryptocurrency market: an evaluation of the performance of momentum strategies in the cryptocurrency market and cryptocurrency's place in an optimized investment portfolio (Master's thesis). Available at: https://openaccess.nhh.no/nhh-xmlui/ bitstream/handle/11250/2487326/masterthesis.PDF?sequence=1. [11.11.2020].

Bollerslev, T. (1986) Generalized Autoregressive Conditional Heteroscedasticity. Journal of Econometrics, 31(6): 307-327. doi:10.1016/0304-4076(86)90063-1.

Bošnjak,M.,Bilas,V.,Novak,I.(2016) Modeling exchange rate volatilities in Croatia. Modeling exchange rate volatilities in Croatia. Ekonomski vjesnik/Econviews-Review of Contemporary Business, Entrepreneurship and Economic Issues, 29(1): 81-94.Available at: https://hrcak.srce.hr/161035 [15.10.2020].

Catania, L., Grassi, S., Ravazzolo, F. (2018) Predicting the volatility of cryptocurrency time-series. Mathematical and Statistical Methods for Actuarial Sciences and Finance, 203-207. Springer, Cham. doi:10.1007/978-3-319-89824-7_37.

Chen, S., Chen, C., Härdle, W. K., Lee, T. M., Ong, B. (2016) A first econometric analysis of the CRIX family. Available at: SSRN 2832099. doi:10.2139/ssrn.2832099 [18.10.2020].

Cheng, L. (2018) Does Bitcoin Offer Diversification Benefit in a Portfolio? (Doctoral dissertation, The Ohio State University). Available at: http://hdl.handle.net/1811/86904 [15.10.2020]. 
Chu, J., Chan, S., Nadarajah, S., Osterrieder, J. (2017) GARCH modelling of cryptocurrencies. Journal of Risk and Financial Management, 10(4): 17. doi:10.3390/jrfm10040017.

Conrad, C., Custovic, A., Ghysels, E. (2018) Long-and short-term cryptocurrency volatility components: A GARCH-MIDAS analysis. Journal of Risk and Financial Management, 11(2): 23. doi:10.3390/jrfm11020023.

Corbet, S., Meegan, A., Larkin, C., Lucey, B., Yarovaya, L. (2018) Exploring the dynamic relationships between cryptocurrencies and other financial assets. Economics Letters, 165: 28-34. doi:10.1016/j. econlet.2018.01.004.

Dhyrberg,A. H. (2016) Hedging capabilities of bitcoin. Is it the virtual gold? Finance Research Letters, 16: 139-144. doi:10.1016/j.frl.2015.10.025.

Ding, Z., Granger, C. W., Engle, R. F. (1993) A Long Memory Property Of Stock Market Returns And A New Model. Journal of Empirical Finance, 1(1): 83-106. doi:10.1016/0927-5398(93)90006-D.

Engle,R.F.(1982) Autoregressive Conditional Heteroscedasticity with Estimates of the Variance of United Kingdom Inflation. Econometrica: Journal of the Econometric Society,50(4): 987-1007.doi:10.2307/1912773.

Glosten, J. R., Jagannathan, R., Runkle, D. E. (1993) On the Relation between the Expected Value and the Volatility of the Nominal Excess Return on Stocks. Journal of Finance, 48(5): 1779-1801. doi:10.1111/j.1540-6261.1993.tb05128.x.

Henriques, I., Sadorsky, P. (2018) Can bitcoin replace gold in an investment portfolio? Journal of Risk and Financial Management, 11(3): 48. doi:10.3390/jrfm11030048.

Higgs, H., Worthington, A. C. (2005) Systematic Features of High-Frequency Volatility in Australian Electricity Markets: Intraday Patterns, Information arrival and Calendar Effects. The Energy Journal, 26(4): 23-42. doi:10.1111/j.1475-4932.2005.00237.x.

Katsiampa,P. (2017) Volatility estimation for Bitcoin: A comparison of GARCH models. Economics Letters, 158: 3-6. doi:10.1016/j.econlet.2017.06.023.

Klaassen, F. (2002) Improving GARCH volatility forecasts with regime-switching GARCH. Advances in Markov-switching models, 223-254. Physica, Heidelberg. doi:10.1007/s001810100100.

Klein, T., Thu, H. P., Walther, T. (2018) Bitcoin is not the New Gold-A comparison of volatility, correlation, and portfolio performance. International Review of Financial Analysis, 59: 105-116. doi:10.1016/j. irfa.2018.07.010.

Letra, I. J. (2016) What drives cryptocurrency value? A volatility and predictability analysis. (Doctoral dissertation, Instituto Superior de Economia e Gestão). Available at: http://hdl.handle.net/10400.5/12556 [15.10.2020].

Nakamoto, S. (2008) Bitcoin: A peer-to-peer electronic cash system. Available at: https://bitcoin.org/ bitcoin.pdf [15.10.2020].

Nelson, D. B. (1991) Conditional Heteroscedasticity in Asset Returns: A New Approach. Econometrica, 59(2): 347-370. doi:10.2307/2938260.

Osterrieder,J.,Lorenz,J.(2017) A statistical risk assessment of Bitcoin and its extreme tail behavior.Annals of Financial Economics, 12(01): 1750003. doi:10.1142/S2010495217500038.

Stavroyiannis, S. (2018) Value-at-risk and related measures for the Bitcoin. The Journal of Risk Finance. doi:10.1108/JRF-07-2017-0115.

Symitsi, E., Chalvatzis, K. J. (2018) Return, volatility and shock spillovers of Bitcoin with energy and technology companies. Economics Letters, 170: 127-130. doi:10.1016/j.econlet.2018.06.012.

Urquhart, A. (2017) Price clustering in Bitcoin. Economics letters, 159: 145-148. doi:10.1016/j. econlet.2017.07.035.

Zakoian,J.M. (1994) Threshold Heteroskedastic Models. Journal of Economic Dynamics and Control, 18(5): 931-952. doi:10.1016/0165-1889(94)90039-6. 
